\title{
HACIA UNA MONEDA DIGITAL EUROPEA. \\ EL EURO 2.0
}

\author{
FRANCISCO HERNÁNDEZ FERNÁNDEZ' \\ Graduate Institute of International \\ and Development Studies \\ francisco.hernandez@graduateinstitute.ch
}

\author{
Cómo citar/Citation \\ Hernández Fernández, F. (2021). \\ Hacia una moneda digital europea. El euro 2.0. \\ Revista de Derecho Comunitario Europeo, 70, 1006-1033. \\ doi: https://doi.org/10.18042/cepc/rdce.70.06
}

\section{Resumen}

La posibilidad de que los bancos centrales emitan dinero digital (CBDC por sus siglas en inglés) ya se está debatiendo. El Banco Central Europeo presentó un informe el 2 de octubre de 2020 sobre la emisión de un euro digital en la eurozona. Es una forma de responder al creciente interés por las criptomonedas, así como de valorar un posible nuevo mecanismo de gestión y control económico. Sin embargo, sus indudables ventajas no aparcan del todo las dudas que aún genera. Este trabajo intenta, de una parte, analizar los CBDC, tradicionalmente confundidos con otros instrumentos que surgen de la digitalización económica, y, de otra parte, estudiar las implicaciones de un CBDC europeo o euro digital, teniendo en cuenta las posibles bases normativas que ofrece el derecho primario de la Unión Europea para su puesta en funcionamiento. Se finalizará estudiando las consecuencias que pueden aflorar de la implantación del euro digital en tres áreas: la política monetaria y fiscal, el sistema de pagos o la propia pervivencia del dinero en efectivo.

\section{Palabras clave}

Política monetaria; euro; euro digital; CDBC; criptomoneda.

1 Becario de la Fundación «la Caixa» en el Graduate Institute of International and Development Studies. LL.M en Droit et Contentieux de l'UE (Université du Luxembourg). 


\title{
TOWARDS A EUROPEAN DIGITAL CURRENCY. THE EURO 2.0
}

\begin{abstract}
The possibility of Central Banks issuing digital money (CBDC) is currently under discussion. The European Central Bank presented a report on October 2, 2020, on the issuance of a digital euro in the Eurozone. It is a way to respond to the growing interest in cryptocurrencies, as well as to assess a possible new management and economic control mechanism. However, its advantages do not entirely dispel the doubts it still generates. This article attempts, on the one hand, to explain what a CBDC is, traditionally confused with other instruments arising from economic digitalization; and on the other hand, to study the implications of a European CBDC or digital euro, considering the possible legal bases available under the primary law of the European Union. This paper will end by studying the consequences that may arise from the implementation of the digital euro in three areas: monetary and fiscal policy, the payment system, and the very survival of cash.
\end{abstract}

\section{Keywords}

Monetary policy; euro; digital euro; CDBC; cryptocurrency.

\section{VERS UNE MONNAIE NUMÉRIQUE EUROPÉENNE. L’EURO 2.0}

\section{Résumé}

La possibilité pour les banques centrales d'émettre de la monnaie numérique (CBDC pour son acronyme en anglais) est actuellement en discussion. La Banque centrale européenne a présenté le 2 octobre 2020 un rapport sur l'émission d'un euro numérique dans la zone euro. C'est une façon de répondre à l'intérêt croissant pour les crypto-monnaies, ainsi que d'évaluer un nouveau mécanisme de gestion et de contrôle économique. Cependant, ses avantages ne dissipent pas entièrement les doutes qu'elle suscite encore. Cet article tente, d'une part, d'expliquer ce qu'est un CBDC, traditionnellement confondue avec d'autres instruments issus de la numérisation de l'économie; et d'autre part, d'étudier les implications d'un CBDC européenne ou d'un euro numérique, en considérant les éventuelles bases juridiques disponibles dans le droit primaire de l'Union européenne. Ce travail conclut en analysant les conséquences qui pourraient en découler de la mise en œuvre de l'euro numérique dans trois domaines: la politique monétaire et fiscale, le système de paiement et la survie même de l'argent liquide.

\section{Mots clés}

Politique monétaire; euro; euro numérique; CDBC; crypto-monnaie. 


\section{SUMARIO}

I. INTRODUCCIÓN. II. HACIA UN EURO DIGITAL COMO MONEDA DE CURSO LEGAL: 1. Los CBDC y los métodos de pago. 2. El proyecto del euro digital: el CBDC de la UE. III. IMPLICACIONES DEL EURO DIGITAL EN LA UNIÓN ECONÓMICA Y MONETARIA: 1. El euro digital y la política monetaria. 2. El euro digital y la política fiscal. 3. El euro digital y el sistema de pagos. 4. El euro digital y la supresión del efectivo. IV. CONCLUSIÓN. BIBLIOGRAFía.

\section{INTRODUCCIÓN}

La Unión Económica y Monetaria (UEM) ha sido uno de los grandes éxitos de la Unión Europea. Aun cuando la actual política monetaria del Banco Central Europeo (BCE) de bajos tipos de interés ha sido duramente criticada por Paul Kirchhof (2021: 215), llegando a ser calificada de inconstitucional por ser contraria al derecho de propiedad privada y a la igualdad ante la ley, pues priva a los ahorradores de las ganancias del capital mientras favorece a los inversores a que pidan prestado dinero. Sin embargo, el euro, la materialización de la política monetaria común y símbolo de la Unión Europea (UE) ${ }^{2}$, goza de un amplio respaldo. En una reciente encuesta ${ }^{3}$ llevada a cabo por la Comisión Europea en los diecinueve países de la eurozona, el $80 \%$ de los participantes creían que el euro era algo positivo para la UE y un $70 \%$ consideraron que lo era para sus respectivos países. La moneda única ha sido incluso adoptada por Estados que no son miembros de la UE, bien mediante acuerdos monetarios, como es el caso de algunos micro-Estados europeos (Ciudad del Vaticano, San Marino, Mónaco y Andorra), bien unilateralmente, como en Kosovo y Montenegro. Además, el euro es la segunda moneda más importante en el sistema monetario internacional ${ }^{4}$.

2 Declaración n. ${ }^{\circ} 52$ al Tratado de Lisboa.

3 European Commission, Directorate-General for Economic and Financial Affairs, y Ipsos European Public Affairs (2021): The Euro Area. Flash Eurobarometer, 3.

4 European Central Bank (2021): The International Role of the Euro, 4. Disponible en: https://bit.ly/3DVEsfu. [Consulta: 6 agosto 2021]. 
Sin embargo, el miedo de los Estados a que una política monetaria europea pudiera derivar en una política económica única acabó resultando en una separación formal: una, competencia exclusiva de la UE, y otra, aún hoy, en manos de los Estados miembros. En la actualidad, el derecho primario obliga solamente a una cierta coordinación en el ámbito de la política económica. De esta manera, el art. 119 del Tratado de Funcionamiento de la UE (TFUE) establece que la política económica se basará en la estrecha coordinación de las políticas económicas de los Estados miembros y en la definición de objetivos comunes. Además, el art. 121 del TFUE señala que los Estados miembros «considerarán sus políticas económicas como una cuestión de interés y las coordinarán en el seno del Consejo». Por su parte, el pilar monetario de la UEM apenas se ha visto alterado desde su adopción en el Tratado de Maastricht de 1992. Pese a ello, la política monetaria se encuentra en pleno proceso de reconfiguración, aunque no se ha llevado a cabo aún ninguna reforma substancial en los Tratados.

En octubre de 2020, el BCE publicó su primer informe sobre el euro digital. Se trataría de una moneda digital del banco central, también conocida como central bank digital currency (CBDC por sus siglas en inglés) que coexistiría eventualmente con la actual moneda física. La puesta en marcha de un CBDC podría tener importantes repercusiones sobre la política monetaria europea e implica una actualización indirecta de las funciones del Sistema Europeo de Bancos Centrales (SEBC) de definir y ejecutarla, así como de promover el buen funcionamiento de los sistemas de pago de acuerdo con el art. 127.2 TFUE. Por otro lado, el 8 de julio de 2021 el Consejo de Gobierno del BCE aprobó su nueva estrategia de política monetaria que contempla un novedoso objetivo de inflación, esta vez del $2 \%$ a medio plazo, y que ha de incluir consideraciones climáticas 5 .

El objetivo de este trabajo es analizar la posible introducción del euro digital en la UE. El trabajo se divide en dos partes. En la primera, analizaremos el panorama actual de los métodos de pago y la aparición de los CBDC, así como el estado actual del proyecto de euro digital a la luz de la reciente jurisprudencia del Tribunal de Justicia de la UE (TJUE). En la segunda, examinaremos las implicaciones que podría tener el euro digital en la UEM, en particular en la política monetaria y fiscal, en el sistema de pagos, así como si la adopción del euro digital conllevaría una supresión del dinero en efectivo.

5 European Central Bank (2021): El Consejo de Gobierno del BCE aprueba su nueva estrategia de politica monetaria. Disponible en: https://bit.ly/3jh5C8C. [Consulta: 14 agosto 2021]. 


\section{HACIA UN EURO DIGITAL COMO MONEDA DE CURSO LEGAL}

\section{LOS CBDC Y LOS MÉTODOS DE PAGO}

El CBDC consiste en dinero digital emitido por el Banco Central Nacional (BCN) en unidad de cuenta nacional, es decir, en la moneda de curso legal y que representa un pasivo del $\mathrm{BCN}^{6}$. Se atribuye su invención al informático estadounidense David Chaum cuando hace más de 35 años ideó una versión electrónica y anonimizada del dinero en efectivo (Auer y Böhme, 2021: 4).

Actualmente la adopción de una moneda digital es uno de los temas más importantes que están siendo abordados por la gran mayoría de BCN del mundo. De acuerdo con una encuesta llevada a cabo a finales de 2020 por el Banco Internacional de Pagos (BIP) (Boar y Wehrli, 2021: 11), entre más de sesenta bancos centrales, el $86 \%$ de los encuestados respondieron que están explorando los beneficios e inconvenientes de implantarla en sus respectivas jurisdicciones, aunque solo el $60 \%$ (Boar y Wehrli, 2021: 11) considera posible hacerlo en el corto y medio plazo.

Algunos $\mathrm{BCN}$ han adoptado proyectos piloto, pero aún ninguno ha sido completamente implementado. Así, por ejemplo, el 20 de octubre de 2020 se puso en circulación en Bahamas el primer CBDC del mundo, el Sand Dollar, para los residentes en el país. También el Banco Central del Caribe Oriental ha lanzado el suyo, el DCash, para Antigua y Barbuda, Granada, Santa Lucía y San Cristóbal y Nieves, con miras a generalizarlo en breve para el resto de los países de la organización. Igualmente, el Banco Nacional de la República Popular de China está llevando a cabo un programa piloto para adoptar el yuan electrónico o e-CNY y el Banco de Suecia se encuentra en una fase avanzada para implementar su e-krona. Otros países como Dinamarca, Noruega, Brasil, Ecuador, Camboya, Islandia, Sudáfrica, Ucrania, Uruguay, Israel y Suiza están también considerando adoptar un CBDC (Auer y Boehme, 2020: 97-98).

En la actualidad existen cinco tipos de pago (Adrian y Mancini-Griffoli, 2019: 3): (1) el dinero del banco central, (2) las criptomonedas, (3) el denominado dinero bancario o b-money, (4) el dinero electrónico o e-money y (5) el i-money, es decir, el dinero de inversión, emitido por fondos de inversión privados. Un CBDC es completamente diferente a todos los métodos de pago existentes, ya que constituye un crédito directo de un $\mathrm{BCN}$ en lugar de un

6 Bank for International Settlements (2021): CBDCs: An Opportunity for the Monetary System. Annual Economic Report, 65. Disponible en: https://bit.ly/3APUohn. 
pasivo de una institución financiera privada como ocurre cuando se realizan transferencias bancarias.

Los CBDC han de ser distinguidos de los métodos de pago con los que generalmente son confundidos. En primer lugar, respecto de las criptomonedas. En ningún caso las criptodivisas o criptomonedas como el bitcoin, ethereum o litecoin son monedas desde el punto de vista legal, puesto que no cuentan con el respaldo de un banco central u otra autoridad pública y no están respaldadas por un mecanismo de protección de depósitos como aquellos constituidos en las entidades de crédito ni protegidas por el Fondo de Garantía de Depósitos o el Fondo de Garantía de Inversores ${ }^{7}$. Además, el valor de las criptomonedas es puramente especulativo y se basa en su supuesta escasez, siendo necesario convertirlas a una moneda de curso legal para poder determinar su valor, puesto que no hay una obligación general de aceptarlas como métodos de $\mathrm{pago}^{8}$. Finalmente también se distingue por las repercusiones que implica para el medioambiente el alto consumo energético preciso para su generación, estimándose que la energía necesaria para producir un bitcoin equivale a 500000 transacciones con tarjeta bancaria (Strauss-Kahn, 2021).

Un CBDC tampoco puede considerarse un tipo de dinero electrónico. Esta clase de método de pago disfruta actualmente de un régimen jurídico propio tanto en el derecho español ${ }^{9}$ como en el derecho europeo ${ }^{10}$. Se define como «todo valor monetario almacenado por medios electrónicos o magnéticos que represente un crédito sobre el emisor y que se emita al recibo de fondos con el propósito de efectuar operaciones de pago» ${ }^{11}$. El dinero electrónico no es emitido por el banco central, sino por quienes tienen la condición de emisores

7 Banco de España y CNMV (2021): Comunicado conjunto de la CNMVy del Banco de España sobre el riesgo de las criptomonedas como inversión. Disponible en: https://bit. ly/3DTInt1.

8 Sentencia de 22 de octubre de 2015, Hedqvist, C-264/14, EU:C:2015:718, apdos. 52-55.

9 Ley 21/2011, de 26 de julio, de dinero electrónico; Ministerio de Economía y Competitividad, Real Decreto 778/2012, de 4 de mayo, de régimen jurídico de las entidades de dinero electrónico.

10 Directiva 2009/110/CE del Parlamento Europeo y del Consejo, de 16 de septiembre de 2009, sobre el acceso a la actividad de las entidades de dinero electrónico y su ejercicio, así como sobre la supervisión prudencial de dichas entidades, por la que se modifican las Directivas 2005/60/CE y 2006/48/CE y se deroga la Directiva 2000/46/ CE (DO L 267, de 10.10.2009, p. 7/17).

11 Art. 1.2, Ley 21/2011, de 26 de julio, de dinero electrónico. 
de dinero electrónico ${ }^{12}$ como entidades de crédito, Correos o el Banco de España (siempre que no actúe en su condición de autoridad monetaria). Esta clase de dinero sirve principalmente para facilitar la realización de pequeños pagos por medios electrónicos seguros y de bajo coste que sustituyen el empleo del papel moneda y las monedas en efectivo (Parra, 2018: 8). Además, al igual que en el caso de las criptomonedas, el empleo del dinero electrónico conlleva la conversión obligatoria al dinero de curso legal. Aunque su uso en Europa no está muy extendido, el dinero electrónico está siendo ampliamente utilizado, como una solución a la débil implantación bancaria, en Asia y África (Alipay y WeChat Pay en China, Paytm en India y M-Pesa en África Oriental).

$\mathrm{El}$ reciente interés de los $\mathrm{BCN}$ por adoptar una moneda digital obedece a distintas razones. Por un lado, la creciente digitalización de la economía requiere disponer de métodos de pagos eficaces que faciliten el comercio en línea. Por otro, la aparición de las criptomonedas podría acabar socavando la confianza fundamental en el dinero (Bindseil, 2021: 172) y perjudicar la transmisión de la política monetaria al afectar a la posición de liquidez de los bancos.

Pese a que el dinero es un bien público, y está inexorablemente vinculado con la soberanía del Estado (Mersch, 2019: 19), algunas grandes empresas tecnológicas han empezado a ofrecer servicios financieros al margen del control de los bancos centrales. Así, por ejemplo, en algunos países como en China (Carstens et al., 2021: 4) las dos grandes empresas tecnológicas Alibaba y Tencent representan actualmente el $94 \%$ de la cuota de mercado de pagos a través de teléfonos móviles gracias a sus aplicaciones de pago electrónico. Del mismo modo, otras multinacionales, como Facebook, pretenden adoptar su propia moneda, una stablecoin, el Diem (anteriormente conocida como Libra). Por otro lado, el hecho de que grandes empresas tecnológicas ofrezcan servicios financieros representa también un reto para la protección de datos de los consumidores puesto que su uso es fundamental para su modelo de negocio ${ }^{13}$, mientras que los $\mathrm{BCN}$ no tienen incentivos para monetizar los datos de los usuarios.

Un CBDC ofrece un gran número de ventajas (Cœuré et al., 2020). En primer lugar, permite el uso de la moneda en aquellos lugares donde el acceso al dinero en efectivo es menor o donde la utilización significativa de criptomonedas podría acabar sustituyéndola. En segundo

Ibid., art. 2.

13 Financial Stability Institute (2021): FSI Insights on policy implementation, no. 36. Big tech regulation: what is going on? 10. Disponible en: https://bit.ly/3napFqb. [Consulta: 11 octubre 2021]. 
lugar, protege la soberanía monetaria y la acción de las políticas públicas al prevenir la constitución de monopolios tecnológicos que impidan la inclusión financiera (Fasquelle, 2021: 181). En tercer lugar, mejora los intercambios transfronterizos, que, por lo general, son lentos y caros ofreciendo un nuevo método de pago más eficaz. En cuarto lugar, aporta un cierto nivel de anonimidad a la hora de realizar pagos electrónicos que, por ahora, no existe en este ámbito.

En la actualidad, las distintas propuestas de CBDC pueden clasificarse (Auer et al., 2020: 18) en cuatro grupos según el tipo de estructura o de diseño: el sistema directo operado por el banco central, que se encargaría de registrar y ejecutar todas las transacciones; un sistema híbrido en el que las entidades intermediarias (presumiblemente entidades bancarias) se encargarían de gestionar los pagos minoristas, mientras que el banco central mantendría un registro de todas las transacciones y operaría la infraestructura técnica de respaldo que le permitiría reiniciar el sistema de pagos si los intermediarios fallasen. Un sistema CBDC, intermediado, similar al sistema híbrido, en el que el banco central tan solo mantendría un registro de algún tipo de transacciones. Y, finalmente, un modelo indirecto o sintético en el que el sistema estaría por completo operado por intermediarios, quienes gestionarían todas las transacciones y tendrían que respaldarlas con créditos con el banco central.

\section{PROYECTO DE EURO DIGITAL: EL CBDC DE LA UE}

El 2 de octubre de 2020, el BCE presentó su informe sobre la emisión de un CBDC en la eurozona, el euro digital. El informe se estructura en seis partes, sin incluir la introducción y los correspondientes anexos. La primera parte está dedicada a señalar las razones que existen, repartidas en dos grupos, para adoptar un euro digital. El primer grupo comprende aquellas que afectan a las funciones básicas del banco central ${ }^{14}$ entre las que se incluye: la necesidad de renovar el dinero bancario tradicional a los retos de la digitalización, la disminución en el uso de dinero en efectivo, la aparición de otras formas de dinero que no están en euros, como instrumento para mejorar la transmisión de la política monetaria o para mitigar los efectos sobre los métodos de pagos ante eventos extremos como pandemias o desastres naturales. El segundo grupo comprende aquellas razones vincu-

14 European Central Bank (2020): Report on a digital euro, 9-14. Disponible en: https:// bit.ly/3aRfu4s. 
ladas con objetivos de la UE como fortalecer el papel internacional del euro o combatir la huella ecológica de los sistemas monetarios y financieros ${ }^{15}$.

La segunda parte del informe está dedicada a los efectos potenciales del euro en el sector bancario, sobre la política monetaria y la estabilidad financiera, el sistema de pagos tanto transfronterizos como pagos al por menor. La tercera parte aborda algunas consideraciones jurídicas sobre el euro digital. Por último, la cuarta y quinta se refiere a cuestiones relacionadas con el diseño y especificaciones técnicas.

Se trata de un texto completo que identifica las grandes áreas que han de ser analizadas para poder adoptar el euro digital. Sin embargo, el documento no termina de concretar cómo podrá implantarse, lo que evidencia que su puesta en marcha aún se encuentra en una fase embrionaria. En especial, dos grandes áreas requerirán de un mayor grado de precisión. De una parte, la base jurídica para que el Eurosistema pueda emitirlo. De otra, un estudio detallado sobre las consecuencias que podrían darse en cuatro áreas: efectos indirectos sobre la política monetaria y la política fiscal, incidencia en el sistema de pagos y, por último, impacto sobre la existencia del dinero en efectivo.

En relación con la elección de la base legal para adoptar el euro digital, dependerá de su diseño y del objetivo que se pretenda lograr. El art. 296 TFUE exige que todos los actos jurídicos de la UE han de ser motivados. Por esta razón, el informe del BCE presta atención a este aspecto ${ }^{16}$ aunque no termina por decantarse por ninguna base jurídica en particular.

En este sentido, si se considera que el euro digital es un instrumento de la política monetaria, similar a las reservas de los bancos centrales, el Eurosistema podría invocar, como base jurídica, el apdo. 2 del art. 127 del TFUE, en relación con la primera frase del art. 20 del Estatuto del SEBC y del BCE. Si, por el contrario, el euro digital se pusiera a disposición de los hogares y otras entidades privadas a través de cuentas abiertas en el Eurosistema, este podría invocar el apdo. 2 del art. 127 del TFUE, en relación con el art. 17 del Estatuto del SEBC y del BCE. Si el euro digital se emitiera como medio de liquidación para tipos de pago específicos, procesados por una infraestructura de pagos concreta, la base jurídica sería más bien el apdo. 2 del art. 127 del TFUE junto con el art. 22 del Estatuto del SEBC y del BCE.

15 Ibid., 14-15.

16 European Central Bank (2020): Report on a digital euro, 24. Disponible en: https:// bit.ly/3G3D9N4. 
Por último, si el euro digital se emitiera como instrumento equivalente a un billete o moneda en efectivo, la más conveniente para su emisión sería la del art. 128 del TFUE en relación con la primera frase del art. 16 de los estatutos del SEBC. Algún sector doctrinal (Grünewald et al., 2021: 39-40) se ha decantado por esta última al señalar que el euro digital se asemeja al concepto de billete establecido en el derecho originario. Sin embargo, empleando una interpretación literal del art. 128 TFUE no se podría llegar a esta conclusión. La disposición indica que «el Banco Central Europeo y los bancos centrales nacionales podrán emitir billetes». El verbo «emitir» ${ }^{17}$ que, de acuerdo con la Real Academia de la Lengua Española, significa producir y poner en circulación papel moneda. Aunque es cierto que los Tratados no prohíben expresamente la emisión de moneda digital y que, tal y como ha establecido el TJUE ${ }^{18}$, cada disposición del derecho de la UE debe ser interpretada a la luz de «su grado de evolución en la fecha en la que debe hacerse aplicación de la disposición de que se trata». Sin embargo, una interpretación sistemática de effet utile $\mathrm{o}$ ut res magis valeat quam pereat del derecho originario no es, en ningún caso, una base jurídica habilitante sino una posible fórmula de interpretación de una norma preexistente.

En este sentido la base jurídica más sólida para adoptar el euro digital es el art. 133 del TFUE que estipula que, con arreglo al procedimiento legislativo ordinario, el Parlamento Europeo y el Consejo establecerán las medidas necesarias para la utilización del euro como moneda única. En el caso Hessischer Rundfunk, el TJUE ${ }^{19}$ ha resaltado la importancia de esta base jurídica para garantizar la unicidad del euro. En esta sentencia se estudia la controversia jurídica que se origina cuando a dos ciudadanos alemanes, los Sres. Johannes Dietrich y Norbert Häring, se les rechaza el 1 de septiembre de 2015 la posibilidad de pagar en efectivo el canon obligatorio a la Radiotelevisión pública del Länder de Hesse (Hessischer Rundfunk). Los demandantes sostuvieron que tanto el derecho nacional alemán (en concreto el art. 14 de la Gesetz über die Deutsche Bundesbank) así como el derecho de la UE, el art. 128.1 del TFUE, establecen una obligación incondicional e ilimitada de aceptar billetes denominados en euros como medio de pago para liquidar deudas monetarias al ser la única moneda de curso legal en la Unión.

17 Asimismo, emplea términos análogos y específicos en francés, émettre; inglés, issue; italiano, emettere; y alemán, berechtigt.

18 Sentencia de 6 de octubre de 1982, CILFIT, C-283/81, EU:C:1982:335, apdo. 20.

19 Sentencia de 26 de enero de 2021, Hessischer Rundfunk, C-422/19, EU:C:2021:63, apdos. $42-43$. 
El TJUE ha aprovechado este caso, en primer lugar, para confirmar ${ }^{20}$ que el mandato de la política monetaria del que dispone el SEBC no se limita a su ejecución operativa, sino que «implica una dimensión normativa destinada a garantizar el estatuto del euro como moneda única». En segundo lugar, que el euro, al ser la moneda de curso legal, «no puede rechazarse a la hora del pago de una deuda denominada en la misma unidad monetaria, por su valor nominal y con efecto liberatorio ${ }^{21}$. Ahora bien, la competencia exclusiva de la Unión en materia de política monetaria no se opone a que los Estados miembros cuya moneda es el euro, en el marco de sus competencias propias (como la organización de su Administración Pública) puedan regular las modalidades de extinción de las obligaciones pecuniarias y, en ocasiones, excepcionar la obligación de aceptar el pago en efectivo ${ }^{22}$; siempre que las medidas adoptadas respeten el principio de proporcionalidad y se encuentren justificadas por motivos de orden público como la seguridad, la lucha contra la delincuencia o el interés de garantizar una organización eficaz de los pagos en la sociedad ${ }^{23}$.

Pese a que según el economista alemán Georg Knapp (1923: 1) el dinero es una creación del derecho, sin embargo, tal y como señaló el abogado general Pitruzzella $a^{24}$, no existe ni en el derecho de la UE ni en la mayoría de los sistemas jurídicos nacionales una definición del concepto de moneda. Esto podría dificultar el proceso legislativo de un CBDC en el derecho de la UE, puesto que no se podría regular una versión digital de la moneda sin contar con una definición previa de lo que se entiende por moneda.

No obstante esta laguna jurídica, el derecho de la UE sí regula con detalle algunos elementos de la moneda única como, por ejemplo, las especificaciones técnicas ${ }^{25} \mathrm{o}$ todo lo relacionado con su puesta en circulación ${ }^{26}$. De este modo, el art. 128.1 del TFUE señala que los billetes emitidos por el BCE y los BCN

20 Sentencia de 26 de enero de 2021, Hessischer Rundfunk, C-422/19, EU:C:2021:63, apdo. 38.

21 Ibid., apdo. 46.

22 Ibid., apdo. 56.

23 Ibid., apdos. 66-69.

24 Conclusiones del abogado general Pitruzzella, Hessischer Rundfunk, C-422/19, EU:C:2020:756, punto 75.

25 Reglamento (UE) n. ${ }^{\circ} 729 / 2014$ del Consejo, de 24 de junio de 2014, relativo a los valores nominales y las especificaciones técnicas de las monedas en euros destinadas a la circulación (DO L 194, de 2.7.2014, p. 1/7).

26 Reglamento (UE) nº 651/2012 del Parlamento Europeo y del Consejo, de 4 de julio de 2012, relativo a la emisión de monedas en euros (DO L 201, de 27.7.2012, p. 135/137). 
serán los únicos billetes de curso legal en la Unión y se reconoce, además, que tan solo el BCE podrá autorizar la emisión de billetes en euros en la Unión. Sin embargo, la producción física de las monedas no se lleva a cabo de una manera centralizada. El art. 128.2 del TFUE otorga la competencia a los Estados miembros para la acuñación de moneda metálica. Por esta razón, son las fábricas nacionales las que elaboran las monedas y los billetes de euro bajo el control del BCE (Gortsos, 2020: 324-326) y su aprobación de las especificaciones técnicas de producción, así como del volumen de monedas y billetes según el país. Por su parte, los BCN están encargados de ponerlos y retirarlos de la circulación, así como de destruir los billetes que se encuentren deteriorados. En este sentido, en caso de adoptar un euro digital, las normas de desarrollo posteriores, como especificaciones técnicas, deberían de adoptarse también mediante el procedimiento legislativo ordinario con base en el art. 133 del TFUE.

\section{IMPLICACIONES DEL EURO DIGITAL EN LA UEM}

La implantación de un CBDC en la UE tendrá importantes implicaciones jurídicas, más allá de la base legal que se emplee para adoptarlo. Ante todo, porque no sería correcto considerarlo como una mera versión digital del euro. Los CBDC son un nuevo método de pago que plantea, por ahora, grandes interrogantes, en especial en relación con los posibles efectos colaterales en tres áreas: la política monetaria y fiscal, el sistema de pagos y respecto a la existencia del dinero en efectivo.

\section{EL EURO DIGITAL Y LA POLÍTICA MONETARIA}

Tal y como ha reconocido recientemente el directorio ejecutivo del Fondo Monetario Internacional ${ }^{27}$, el dinero digital tiene grandes implicaciones para el sistema monetario internacional y para la estructura y estabilidad de las economías nacionales.

Un CBDC amplía el abanico de instrumentos de la política monetaria (Bindseil, 2020: 6), por ejemplo, al permitir que el SEBC pueda fijar distintos tipos de interés para el euro físico y el euro digital favoreciendo con una moneda el ahorro y con la otra la inversión. Este ensanchamiento de los

27 IMF (2021): The Rise of Public and Private Digital Money-A Strategy to Continue Delivering on the IMF's Mandate. IMF Policy Paper. Disponible en: https://bit. ly/2Z13Rpj. [Consulta: 8 agosto 2021]. 
instrumentos de la política monetaria no podría venir en un mejor momento. $\mathrm{Si}$, tal y como aseguran algunos economistas (Beckmann et al., 2021: 6), los tipos de interés negativos tuvieron mayor impacto cuando se introdujeron que ahora y fuese cierto también que los bajos tipos de interés están afectando a la rentabilidad de los bancos (Bonatti et al., 2021: 22), lo que fomenta la existencia de grandes bancos en demérito de los pequeños que no pueden asumir los costes económicos, entonces la lógica general nos sugiere que sería conveniente la normalización de los tipos de cambio del dinero.

Aunque ello no estaría exento de riesgos, ya que se podría producir lo que los economistas han designado como la ley de Gresham. Es decir, cuando en un país circulan simultáneamente dos tipos de monedas de curso legal y una de ellas es percibida por el público como «buena» y la otra como «mala», esta última acaba siempre siendo depreciada y da lugar a un doble precio de los bienes y servicios. Algunos economistas (Brunnermeier et al., 2019: 10) auguran que la ley de Gresham también se aplicaría en el caso de las monedas digitales. Por el contrario, de acuerdo con otros expertos es poco probable que afecte a la transmisión de la política monetaria de forma significativa (Mancini-Griffoli et al., 2018: 4).

Por otro lado, debido a que no existe actualmente en el derecho primario una definición precisa de la política monetaria $\operatorname{común}^{28}$, la incidencia de un CBDC podría quedar mitigada. Tal y como ha confirmado el TJUE ${ }^{29}$ en repetidas ocasiones, los Tratados no definen la política monetaria común, pero sí establecen tanto sus objetivos como los medios de que dispone el SEBC para ejecutar dicha política.

Algunas de las funciones monetarias y de operaciones del SEBC comprenderían (López Escudero, 2017: 357), entre otras, la apertura de cuentas en el BCE y en los bancos centrales nacionales a entidades de crédito o a entidades públicas (art. 17 del Estatuto del SEBC y del BCE), las operaciones en mercado abierto tanto de cambio como crediticias (art. 18 del Estatuto del SEBC y del $\mathrm{BCE})$, el establecimiento de reservas mínimas para las entidades de crédito establecidas en los Estados miembros (art. 19 del Estatuto del SEBC y del $\mathrm{BCE}$ ) o la realización de determinadas operaciones financieras exteriores (art. 23 del Estatuto del SEBC y del BCE). La incorporación de un CDBC como instrumento de control monetario podría ser aprobado por el Consejo de

28 Conclusiones de la abogada general Kokott, Pringle, C-370/12, EU:C:2012:675, punto 78 .

29 Sentencias de 27 de noviembre de 2012, Pringle, C-370/12, EU:C:2012:756, apdo. 53; de 16 de junio de 2015, Gauweiler y otros, C-62/14, EU:C:2015:400, apdo. 42; y de 11 de diciembre de 2018, Weiss y otros, C-493/17, EU:C:2018:1000, apdo. 50. 
Gobierno del BCE por mayoría de dos tercios de los votos en virtud del art. 20 del Estatuto del SEBC y del BCE, lo que ayudaría a perfilar la relación del euro digital como un instrumento de la política monetaria.

\section{EL EURO DIGITAL Y LA POLÍTICA FISCAL}

Un euro digital podría acabar teniendo influencia en la política fiscal si, por ejemplo, a través de él se transfiriera dinero a consumidores y empresas. Esto podría ser beneficioso para reforzar la tímida unión económica en caso de crisis. Mediante un CBDC, el BCE podría adoptar un programa de estímulo fiscal para apoyar el consumo a través del efectivo digital y desaparecer después de un corto período. Esta peculiaridad evidencia que se trata de dinero programable (Nabilou, 2020: 307) y no simplemente de una forma tecnológicamente más avanzada de billete o de moneda. Por vez primera, las autoridades tendrán acceso directo y real a los bolsillos de los ciudadanos, lo que podría ser muy relevante en términos económicos.

La moneda digital sería el medio a través de la cual llevar a cabo el denominado "dinero de helicóptero", teorizado por Milton Friedman en 1969. Es decir, el banco central repartirá dinero de manera excepcional entre los ciudadanos como si un helicóptero dejara caer desde el cielo dinero que sería recogido apresuradamente por los miembros de la comunidad convencidos de que se trata de un acontecimiento único e irrepetible. La idea nunca ha sido puesta en práctica y sigue siendo muy controvertida entre los economistas (Dowd, 2018) que alertan de que serviría para fomentar la inflación o permitir que los gobiernos incurran en déficits excesivos.

Se plantea (Larue et al., 2020: 8-9) que esta opción sería mejor que la monitorización de los déficits fiscales que se producen cuando los bancos centrales adquieren deuda pública en los mercados primarios. Aunque el SEBC tiene prohibido en virtud del art. 123 TFUE la financiación monetaria a favor de los Estados, sí está facultado para adquirir bonos soberanos en los mercados secundarios, lo que no puede equiparse a una medida de asistencia financiera a un Estado miembro, tal y como avaló en su momento el TJUE ${ }^{30}$. Por tanto, esta función de un CBDC, incluso en el actual marco jurídico restrictivo de la UEM, no podría descartarse de plano. Convertir el euro digital en un instrumento híbrido de política monetaria y fiscal podría resultar útil para combatir la futura posible deflación o disminución generalizada de los precios en la eurozona. En virtud del nuevo objetivo de inflación simétrico aprobado el 8

30 Sentencia de 16 de junio de 2015, Gauweiler y otros, C-62/14, EU:C:2015:400, apdo. 103 . 
de julio de 2021 por el Consejo de Gobierno del BCE, la deflación debería combatirse de manera más contundente, y un euro digital podría facilitar la transmisión de la política monetaria y cumplir con el objetivo primario de estabilidad de precios. Nótese que, a pesar de lo que se pensó inicialmente, el principal problema del euro durante sus veinte primeros años ha sido la deflación y no la inflación (Gros y Alcidi, 2019: 6).

Según algunos economistas (Bjerg, 2017: 48) y algunos $\mathrm{BCN}^{31}$, si un $\mathrm{CBDC}$ se convirtiera en un instrumento de política fiscal, la distinción entre las intervenciones de política monetaria y de política fiscal acabarían desapareciendo. Esta eventual colisión obligaría a una mayor concertación entre el Ministerio de Hacienda y el BCN. Sin embargo, a nivel europeo la diferencia es clave. Si quedara demostrado que el euro digital podría interferir en la política monetaria o convertirse en un instrumento de política fiscal europea, esto implicaría que el euro digital traspasaría el actual ámbito competencial de la UEM y, por tanto, sería contrario al derecho de la UE.

Ahora bien, aun cuando ello pudiera ser cierto, las posibles ventajas que aportaría un CBDC podrían convencer a los Estados miembros para modificar los Tratados, mediante el procedimiento de revisión ordinario establecido en el art. 48 TUE, y así dar cobertura legal a este nuevo instrumento que conectaría la política monetaria europea con la, hasta ahora, política fiscal de los Estados miembros, reforzaría la UEM y, en definitiva, el proyecto de integración europeo ${ }^{32}$. Algunos Estados miembros como Francia y España han señalado que la adopción de un euro digital contribuiría a reforzar la autonomía estratégica y financiera de la $\mathrm{UE}^{33}$. Por esta razón, es posible intuir que el proyecto del euro digital cuenta ya con algunos apoyos entre los Estados miembros que asumen sus implicaciones económicas.

\section{EL EURO DIGITAL Y EL SISTEMA DE PAGOS}

La implantación de un euro digital supondría grandes cambios en el sector bancario, ya que se podría transferir dinero online sin comisiones, de manera instantánea, a través de una interfaz o plataforma web sin necesidad

31 European Central Bank (2020): Report on a digital euro, 7. Disponible en: https://bit. ly/3BYfzyT.

32 Francia y España han señalado que la adopción de un euro digital contribuiría a reforzar la autonomía estratégica y financiera de la Unión Europea. Véase: Ministerio de Asuntos Económicos y Transformación Digital (2021): Cumbre franco-española. Declaración conjunta sobre asuntos económicos y financieros. Disponible en: https://bit. ly/31ScNWv. [Consulta: 22 agosto 2021].

33 Véase nota 30. 
de disponer de una cuenta bancaria. Esto provocaría un gran cambio en los sistemas de pago en la eurozona, y en especial en la Zona Única de Pagos en euros (SEPA por sus siglas en inglés), que en la actualidad permite a los consumidores efectuar pagos en euros desde una cuenta bancaria a cualquier otra situada en un país que participe en la SEPA y en las mismas condiciones como si se tratase de un pago nacional. Si bien ello supondría un reto para el modelo actual de negocio de las entidades bancaria, tal y como señala el profesor Hinojosa (2021: 498), el art. 127 TFUE establece un límite legal a la actuación del BCE en el sentido de que la fórmula de implantación del euro digital no puede suponer la desaparición de la banca comercial.

Tal vez sería irreal, de acuerdo con el estado actual de la tecnología, imaginar la implantación de un sistema directo. Todo parece indicar que el sector privado gestionaría mejor ciertas tareas como la identificación del consumidor, la resolución de litigios, la gestión de pagos minoristas, el control del blanqueo de capitales y la financiación del terrorismo o la de informar a la administración tributaria. Esta misma opinión aparece en la consulta pública que el BCE decidió llevar a cabo con ocasión de la publicación del informe del euro digital ${ }^{34}$, entre el 12 de octubre de 2020 y el 12 de enero de 2021, para conocer las inquietudes tanto de profesionales como de ciudadanos. Entre las 8221 respuestas recibidas, el $73 \%$ se decantó por la opción en la que los intermediarios se encargasen de garantizar la interfaz para efectuar los pagos ${ }^{35}$, pero que no implicase costes adicionales por su utilización.

Sin embargo, el derecho actual de la UE se opondría igualmente a un sistema indirecto o sintético de CBDC, operado por completo por intermediarios. En primer lugar, de acuerdo con la doctrina Meroni elaborada por el $\mathrm{TJUE}^{36}$, una institución europea no puede delegar "poderes discrecionales que impliquen un amplio margen de discrecionalidad». Es decir, que los poderes delegados deben estar exactamente definidos y enteramente controlados por la institución que los delega. En este supuesto, los intermediarios bancarios no podrían en ningún caso gestionar el euro digital como si se tratase de un servicio financiero cualquiera; de lo contrario, se pondría en riesgo la unicidad de la moneda única, ya que en la práctica cada banco regularía de manera distinta la utilización del euro digital y se pervertiría la razón de ser de un CBDC.

34 European Central Bank (2021): Eurosystem Report on the Public Consultation on a Digital Euro. Disponible en: https://bit.ly/3AQxEO1.

35 European Central Bank (2021): Eurosystem Report on the Public Consultation on a Digital Euro, 19. Disponible en: https://bit.ly/3nadWIi.

36 Sentencia de 13 de junio de 1958, Meroni, C-9/56, EU:C:1958:7, apdo. 153. 
En segundo lugar, debido a los bajos tipos de interés del dinero es inevitable pensar que algunos intermediarios bancarios desearían poder cobrar a los consumidores por el uso del euro digital. Sin embargo, siempre que el BCE se encargase de crear y mantener la pasarela para efectuar pagos en euros digitales, las entidades no podrían cobrar una comisión por su uso, puesto que un $\mathrm{CBDC}$ representa un pasivo del BCN y serían los intermediarios los que tendrían que pagar al BCE por comercializarlos. Además, el art. 19 de la Directiva sobre los derechos de los consumidores ${ }^{37}$ establece que «los Estados miembros prohibirán a los comerciantes cargar a los consumidores por el uso de determinados medios de pago, comisiones que superen el coste asumido por el comerciante por el uso de tales medios». Es decir, en caso de que el BCE no decidiera cobrar a los intermediarios por distribuir el euro digital, estos no podrían exigir el pago de ninguna comisión. Además, respecto de las posibles comisiones por el servicio de intermediación, el art. 62.5 de la Directiva europea sobre servicios de pago ${ }^{38}$ señala que «los Estados miembros podrán prohibir o limitar el derecho del beneficiario a cobrar gastos teniendo en cuenta la necesidad de fomentar la competencia y promover el uso de instrumentos de pago eficientes».

En definitiva, el modelo de diseño que tendría más posibilidades de implantarse en la eurozona sería un sistema híbrido en el que las entidades intermediarias se encargarían de gestionar pagos minoristas, mientras que el BCE se encargaría de mantener un registro general de transacciones y una interfaz digital que serviría de pasarela para efectuar las transacciones y evitar el cobro de cualquier comisión por parte de los intermediarios. Aunque esto supondría ${ }^{39}$ que los $\mathrm{BCN}$ acabarían siendo responsables del diseño del sistema en su conjunto y en el que se produciría un reparto de roles entre entidades

37 Directiva 2011/83/UE del Parlamento Europeo y del Consejo, de 25 de octubre de 2011, sobre los derechos de los consumidores, por la que se modifican la Directiva 93/13/CEE del Consejo y la Directiva 1999/44/CE del Parlamento Europeo y del Consejo y se derogan la Directiva 85/577/CEE del Consejo y la Directiva 97/7/CE del Parlamento Europeo y del Consejo (DO L 304, de 22.11.2011, p. 64/88).

38 Directiva (UE) 2015/2366 del Parlamento Europeo y del Consejo de 25 de noviembre de 2015 sobre servicios de pago en el mercado interior y por la que se modifican las Directivas 2002/65/CE, 2009/110/CE y 2013/36/UE y el Reglamento (UE) no 1093/2010 y se deroga la Directiva 2007/64/CE (DO L 337, de 23.12.2015, p. 35/127).

39 Bank for International Settlements (2021): Central bank digital currencies: system design and interoperability, 4. Disponible en: https://bit.ly/3phIRW2. [Consulta: 11 octubre 2021]. 
públicas y privadas que exigiría el desarrollo de una serie de normas jurídicas de acompañamiento.

En este sentido, algunos miembros destacados del $\mathrm{BCE}^{40}$ han dado a entender que el euro digital se emplearía para pequeños pagos y que se establecería un límite de euros digitales por persona para evitar la especulación en tiempos de crisis. Es decir, que se establecerían umbrales que fijarían el número de euros digitales que un usuario puede tener. Sin embargo, esto plantea grandes interrogantes sobre qué ocurriría en caso de que se sobrepasase el límite. Se ha sugerido (Bindseil, 2021: 175) que la demanda podría controlarse mediante algún sistema de incentivo en virtud del cual se aplicaran tipos de interés o comisiones de servicio menos atractivos cuando se superara el umbral mencionado. Esto tendría la ventaja de permitir a los usuarios decidir la cantidad de euros digitales que deseen mantener en relación con otras formas de inversión. También se podría establecer que cualquier euro digital entrante que supere el límite de tenencia se trasladaría automáticamente a la cuenta del beneficiario en dinero privado. En cualquier caso, estas opciones dependerán del grado de implantación y de aceptación que tuviese el euro digital en la economía y precisarán que el $\mathrm{BCE}$ controle en todo caso la tecnología necesaria para permitir el pago en euros digitales.

Otra cuestión, también relevante, es decidir qué tecnología emplear en la liquidación de pagos. Por un lado, se duda si emplear blockchain, que, por ejemplo, el Banco Europeo de Inversiones ha comenzado a usar ${ }^{41}$, o si emplear el instrumento de liquidación de pagos inmediatos de TARGET (TIPS por sus siglas en inglés) que el BCE puso en marcha en 2018 (Athanassiou, 2020: 732) y que ofrece a las entidades bancarias la posibilidad de liquidar operaciones de pago individuales en menos de diez segundos utilizando dinero de banco central. De acuerdo con un estudio reciente del BCE, ${ }^{42}$ con una solución basada en cadena de bloques, el $95 \%$ de las transacciones podrían ser firmadas por el pagador, liquidadas y verificadas criptográficamente por el beneficiario en menos de tres segundos, mientras que empleando el TIPS, el mismo porcentaje de transacciones podrían liquidarse con una latencia de

40 European Central Bank (2021): Interview with Financial Times Interview of the Financial Times with Fabio Panetta, Member of the Executive Board of the ECB. Disponible en: https://bit.ly/3DSzIHl.

41 Banque Européenne d'investissement (2021): La BEI émet sa toute première obligation numérique sur une chaîne de blocs publique. Disponible en: https://bit.ly/3GX7k9k. [Consulta: 8 agosto 2021].

42 European Central Bank (2021): Digital Euro experimentation scope and key learnings, 4. Disponible en: https://bit.ly/2ZceK7v. [Consulta: 11 octubre 2021]. 
menos de 0,8 segundos. En todo caso, el diseño que se adopte para gestionar las transacciones del euro digital ha de ser lo suficientemente estable y fiable como para garantizar un gran número de pagos por segundo. El Innovation Hub del BPI se encuentra actualmente investigando la viabilidad tecnológica de los CBDC y su eficacia para distintos fines. Así, el proyecto Helvetia está analizando la viabilidad de los CBDC para gestionar pagos a gran escala. Y el Innovation Hub del BPI ${ }^{43}$ está explorando, tanto en el proyecto Dunbar como en el proyecto Jura, la posibilidad de intercambiar CBDC en distintas monedas, lo que podría contribuir a mejorar las transacciones transfronterizas que actualmente son costosas y lentas. Los resultados de estas investigaciones serán decisivos para determinar no solo la viabilidad real de los CBDC sino también la tecnología necesaria para implementar el euro digital.

\section{EL EURO DIGITAL Y LA SUPRESIÓN DEL EFECTIVO}

En principio, el euro digital sería un complemento del dinero en efectivo $^{44}$ y por ahora no se baraja su desaparición. En sus conclusiones, el abogado general Pitruzzella, a diferencia de lo que luego recogió el TJUE en la sentencia, menciona expresamente ${ }^{45}$ la posibilidad de que en el ámbito europeo se adopte una moneda digital, aunque se muestra escéptico acerca de la aparición de una sociedad sin efectivo (cashless society). De hecho, argumenta ${ }^{46}$, siguen existiendo en la UE personas de sectores vulnerables de la sociedad que aún no tienen acceso a servicios financieros básicos y para quienes el efectivo constituye la única forma disponible de dinero. Además, alerta $^{47}$, que corresponde al BCE, en el marco de su función consultiva (arts. 127.4 TFUE y 282.5 TFUE), y a la Comisión Europea, como guardiana de los Tratados a través de los procedimientos de infracción, asegurar la compatibilidad de las normas nacionales que restrinjan el uso de efectivo como medio de pago.

La situación preconizada por el abogado general ya se ha hecho realidad con ocasión de la consulta que el Banco Nacional de Polonia realizó el 21

43 Bank for International Settlements et al. (2021): Central Bank Digital Currencies for Cross-Border Payments, 25-28. Disponible en: https://bit.ly/3kd8ST2 [Consulta: 1 agosto 2021].

44 European Central Bank (2020): Report on a Digital Euro, 3. Disponible en: https:// bit.ly/3aOS27V.

45 Conclusiones del abogado general Pitruzzella, Hessischer Rundfunk, C-422/19, EU:C:2020:756, punto 82.

46 Ibid., puntos 136-137.

47 Ibid., punto 139. 
de abril de 2021 al BCE respecto al proyecto de ley de servicios de pago que obligaría a emplear únicamente dinero en efectivo en casos de transacciones de bienes y servicios entre comerciantes y consumidores. Si bien la obligación que establecería la norma nacional no sería en ningún caso absoluta, ya que el proyecto de ley preveía algunas excepciones a esta obligación en caso de que la transacción se llevase a cabo de manera electrónica, el establecimiento no disponga de personal presente en la tienda o en eventos multitudinarios. El 14 de mayo de 2021, el BCE emitió su opinión basándose ${ }^{48}$ en la sentencia del TJUE, caso Hessischer Rundfunk, y avaló el proyecto de ley polaco al señalar que los Estados miembros pueden adoptar cualquier medida que apoye el estatus del euro como moneda de curso legal siempre que las medidas sean proporcionales.

Cabría pensar que el proyecto de ley polaco de establecer la obligatoriedad de emplear dinero en efectivo va a contracorriente de los tiempos. Sin embargo, el pago en efectivo es la norma en muchos países de la UE. Así, por ejemplo, el Tribunal Supremo de Alemania estableció en 1986 que las obligaciones monetarias han de realizarse en efectivo a menos que las partes lo acuerden de otro modo (Arnold, 2020: 146). Este mismo enfoque es el que preconiza el derecho español, puesto que, salvo acuerdo previo de las partes, el art. 1.170 del Código Civil estipula que «el pago de las deudas de dinero deberá hacerse en la especie pactada y, no siendo posible entregar la especie, en la moneda de plata u oro que tenga curso legal en España».

De otra parte, Siekmann (2020: 49) llega incluso a afirmar que la supresión de la moneda en efectivo a nivel nacional sería contaria al derecho de la UE porque de conformidad con el art. 128 TFUE «los billetes emitidos por el Banco Central Europeo y los bancos centrales nacionales serán los únicos billetes de curso legal en la Unión». Sin embargo, en principio, un CBDC no es estrictamente una moneda de curso legal, es decir, podría emitirse el euro digital sin que tuviera esta naturaleza. Esta opción teórica plantea algunas dificultades prácticas (Papapaschalis, 2021: 205). Si se determinara que el euro digital es una moneda de curso legal esto afectaría al diseño del CBDC y, para garantizar su aceptación obligatoria, su tecnología tendría que ser compatible con todos los programas y equipos informáticos utilizados para realizar pagos. Además, tampoco sería posible establecer un

48 European Central Bank (2021): Opinion of the European Central Bank of 14 May 2021 on the obligation to accept cash payments, 5. Disponible en: https://bit.ly/3pbp$\mathrm{PjY}$. 
límite de euros digitales por persona para evitar la especulación puesto que esto no existe en la actualidad en el caso del euro físico.

Por otro lado, König (2017: 353) indica que una eventual obligación de utilizar sistemas de pago que no fueran en efectivo perjudicaría su privacidad y anonimato, así como supondría una violación generalizada a la protección de datos de los ciudadanos. Incluso, frente a los supuestos beneficios de transparencia que aporta los nuevos métodos de pago, señala (König, 2017: 344-348) que en aquellos países donde existen limitaciones al pago en efectivo a partir de un determinado umbral (que oscilan desde los 1000 euros en Portugal o Francia a los 3000 euros en Bélgica) hay mayor corrupción que en aquellos donde es más elevado o no existe un límite establecido.

Con independencia del lógico contraste de mentalidades «Norte-Sur», lo cierto es que la preferencia de usar moneda en efectivo, en vez de emplear otros métodos de pago, obedece a razones culturales propias de la idiosincrasia de cada país. De acuerdo con el $\mathrm{BCE}^{49}$, en 2016 el pago en efectivo fue el método predominante en los Estados que han adoptado el euro. El $79 \%$ de todas las transacciones de la eurozona se realizaron en efectivo y, en especial en los países del sur de Europa, además de en Alemania, Austria y Eslovenia, el $80 \%$ de transacciones se llevaron a cabo en efectivo. Sin embargo, en Países Bajos, Estonia y Finlandia el pago en efectivo representaba solamente entre el $45 \%$ y el $54 \%$.

Incluso antes de la crisis sanitaria causada por la COVID-19, los hábitos de pago de los consumidores europeos empezaron a cambiar. De acuerdo con una encuesta llevada a cabo en 2019 por el $\mathrm{BCE}^{50}$, el $73 \%$ de los pagos realizados se efectuaron con dinero en metálico, algo menos que en 2016. La pandemia ha supuesto que el pago en metálico, incluso en países donde tradicionalmente estaba muy extendido, se haya reducido de manera significativa. Así, en 2020 tan solo el $60 \%$ de las compras efectuadas en Alemania se realizaron empleando dinero en efectivo ${ }^{51}$. A esto se añade la innegable generalización del uso de smartphone o de internet por parte de los jóvenes. Según Eurostat, en 2016 casi ocho de cada diez usuarios de internet en la UE

49 European Central Bank (2017): The Use of Cash by Households in the Euro Area, 4. Disponible en: https://data.europa.eu/doi/10.2866/377081.

50 European Central Bank (2020): Study on the Payment Attitudes of Consumers in the Euro Area (SPACE), 5. Disponible en: https://bit.ly/3DYeGXE.

51 Deutsche Bundesbank (2021): Making Payments in 2020, the Year of COVID-19: Card-Based and Contactless Payments Trending. Disponible en: https://bit.ly/2Z0Zt$\mathrm{pU}$. 
lo hacían a través del teléfono móvil ${ }^{52}$, mientras que en $2019^{53}$ el $77 \%$ de la población utilizaba diariamente internet y en el caso de los jóvenes la cifra ascendía a un $94 \%$. En principio, los estudios recientes ${ }^{54}$ se decantan a favor de que el CBDC se implante a través de tecnología ya en uso por los usuarios, como un smartphone, aunque no puede descartarse que se pueda adoptar un hardware específico ${ }^{55}$ que pudiera no necesitar conectarse a internet.

En general, el dinero en metálico ofrece ventajas con las que una CBDC no podrá competir, en especial respecto al anonimato que proporciona. De la consulta pública sobre el euro digital ${ }^{56}$ se desprende que la privacidad es la principal característica que ha de cumplir. Como antes hemos indicado, el empleo de tecnología blockchain podría garantizar cierto anonimato, no de forma absoluta ${ }^{57}$, si bien habría de evitarse la financiación de actividades ilícitas y el lavado de dinero.

Por otro lado, conviene tener en cuenta que el dinero en efectivo tiene también grandes desventajas. En primer lugar, facilita la economía sumergida, tal y como señala el derecho derivado ${ }^{58}$. Se cree que por esta razón, el BCE decidió en 2016 dejar de imprimir los billetes de 500 euros, aunque también podría interpretarse como una maniobra para mantener unos tipos de interés negativos al penalizar que se acumule dinero en forma de reservas en los bancos (Murphy, 2016: 348). En segundo lugar, el dinero en efectivo no es posible emplearlo de manera directa en el comercio electrónico. En tercer

52 Eurostat (2016): Almost 8 out of 10 internet users in the EU surfed via a mobile or smart phone in 2016. Disponible en: https://bit.ly/3BZ7R7L.

53 Eurostat (2021): Being Young in Europe Today. Digital World. Disponible en: https:// bit.ly/3m1YFKx.

54 Bank for International Settlements, 2021. Central bank digital currencies: user needs and adoption, 3. Disponible en: https://bit.ly/3Ee30As. [Consulta: 11 octubre 2021].

55 Deutsche Bundesbank (2021):. Eurosystem experimentation regarding a digital euro Research workstream on hardware bearer instrument. Disponible en: https://bit.ly/3aRV0Zc. [Consulta: 11 octubre 2021].

56 European Central Bank (2021):. Eurosystem Report on the Public Consultation on a Digital Euro, 3. Disponible en: https://bit.ly/2XrGzYP.

57 OECD (2019): OECD-Blockchain-Primer. Disponible en: https://bit.ly/3bRdLMS. [Consulta: 3 agosto 2021].

58 Considerando n. ${ }^{\circ} 6$ de la Directiva (UE) 2015/849 del Parlamento Europeo y del Consejo, de 20 de mayo de 2015, relativa a la prevención de la utilización del sistema financiero para el blanqueo de capitales o la financiación del terrorismo, y por la que se modifica el Reglamento (UE) n. ${ }^{\circ}$ 648/2012 del Parlamento Europeo y del Consejo, y se derogan la Directiva 2005/60/CE del Parlamento Europeo y del Consejo y la Directiva 2006/70/CE de la Comisión (DO L 141, de 5.6.2015, p. 73/117). 
lugar, en caso de insolvencia de un banco, el acreedor solo tiene asegurado hasta 100000 euros en dinero en metálico de acuerdo con las disposiciones europeas de garantía de depósitos ${ }^{59}$, mientras que el euro digital plantearía otro escenario al ser directamente un pasivo del SEBC. En cuarto lugar, en algunos países como Suecia, México, Noruega o Dinamarca los ingresos públicos por acunación de moneda (el denominado señoreaje) se encuentran cercanos a cero (Arvidsson, 2018: 88). Es decir, si esta tendencia continuase, los $\mathrm{BCN}$ tendrían que asumir unos costes por acuñar moneda en metálico. Al mismo tiempo, si un CBDC condujera a un menor uso de efectivo, los bancos podrían reducir los costes asociados al manejo de efectivo, ayudando a su rentabilidad general ${ }^{60}$. Se ha estimado que las operaciones de efectivo representan entre el $5 \%$ y el $10 \%$ de los costes operativos totales de los bancos. Un CBDC podría contribuir a solucionar estos problemas a los que actualmente se enfrenta el dinero en efectivo.

A diferencia de lo que ha argumentado algún autor (Lupo-Pasini, 2021: 33), la supresión del dinero en metálico no se decidirá teniendo en cuenta el nivel de exclusión social que estamos dispuestos a aceptar en nuestra sociedad, sino más bien en base a un criterio utilitarista. Al igual que los teléfonos móviles han ido poco a poco remplazando la utilidad de las cabinas de teléfono. No es ilógico pensar que en un futuro próximo un CBDC que tuviera un diseño eficiente y fácil de usar acabaría remplazando irremediablemente el dinero en efectivo. El euro digital no debería de ser una excepción.

\section{CONCLUSIÓN}

El CBDC supone una evolución del dinero en metálico y es un instrumento necesario para permitir la digitalización de la economía al tiempo que se garantiza la soberanía monetaria de los Estados. Nueve meses después desde la publicación del informe del BCE sobre el euro digital, su Consejo de Gobierno decidió, el 14 de julio de $2021^{61}$, iniciar finalmente la fase de investigación del proyecto. Se prevé que esta nueva

59 Art. 6 Directiva 2014/49/UE del Parlamento Europeo y del Consejo, de 16 de abril de 2014, relativa a los sistemas de garantía de depósitos (DO L 173, de 12.6.2014, p. 149/178).

60 Bank for International Settlements (2021): Central bank digital currencies: financial stability implications, 9. Disponible en: https://bit.ly/3Ee30As. [Consulta: 11 octubre 2021]

61 European Central Bank (2021): El Eurosistema pone en marcha el proyecto de un euro digital. Disponible en: https://bit.ly/3G2Bx6w. 
etapa dure unos veinticuatro meses y estará dedicada a abordar aspectos clave relativos al diseño y la distribución de la moneda digital.

Esta fase es necesaria en parte porque la solicitud de registro de marca comunitaria de euro digital sigue aún pendiente de aprobación por parte de la Oficina de Propiedad Intelectual de la Unión Europea ${ }^{62}$. También se espera que durante ese periodo se determinen las modificaciones legislativas necesarias para llevar a cabo la materialización del proyecto. Igualmente, se evaluarán durante este tiempo las posibles repercusiones de un euro digital en el conjunto de la economía de la zona euro. El proyecto de euro digital tiene todavía por tanto que ser definido, aunque se podría optar por un modelo híbrido en el que se establezca una distribución de tareas entre el sector público y el privado.

Respecto a la base jurídica, el SEBC todavía tiene que determinar las repercusiones que tendría la adopción del euro digital y elegir en consecuencia entre las distintas opciones disponibles en el derecho primario de la UE. El art. 133 TFUE ofrece una solución óptima tanto para la adopción del euro digital como para las futuras normas de acompañamiento que establezcan las especificaciones técnicas necesarias para su puesta en marcha.

El euro digital ampliará los instrumentos de la política monetaria común, si bien no está claro si puede acabar colisionando con la política fiscal, aún en manos de los Estados miembros. Asimismo, también se desconoce su posible impacto sobre el sistema de pagos de la zona euro y si en el largo plazo el dinero en efectivo acabará desapareciendo pese a lo previsto en el art. 128 TFUE. Si finalmente quedara demostrado que el euro digital puede originar interferencias en la transmisión de la política monetaria, o convertirse en un instrumento de política fiscal, esto requería de una reforma del derecho primario. Aunque actualmente el euro digital todavía se encuentra en una fase embrionaria, la aparición de un CBDC en el derecho de la UE tiene el potencial de renovar la UEM.

\section{Bibliografía}

Adrian, T. y Mancini-Griffoli, T. (2019). The Rise of Digital Money. International Monetary Fund. Annual Review of Financial Economics, 13. Disponible en: https://bit.ly/3jgJGKK.

62 European Union Intellectual Property Office: Trade mark information 018311625 Digital Euro. Disponible en: https://bit.ly/2ZcK6uG. 
Arnold, S. (2020). The Euro in German (private) law - monetary obligations and the mutual dependence of public and private law. En R. Freitag y S. Omlor (eds.). The Euro as Legal Tender (pp. 141-160). Berlin: De Gruyter. Disponible en: https://doi:10.1515/9783110640717-007.

Arvidsson, N. (2018). The future of cash. En R. Teigland, S. Siri, A. Larsson, A. M. Puertas y C. I. Bogusz (eds.). In The Rise and Development of FinTech: Accounts of Disruption from Sweden and Beyond (pp. 85-98). London: Routledge. Disponible en: https://doi:10.4324/9781351183628.

Athanassiou, P. L. (2020). Payment Systems. En F. Amtenbrink, C. Herrmann y R. Repasi (eds.). The EU Law of Economic and Monetary Union (pp. 711-735). Oxford: Oxford University Press. Disponible en: https://doi:10.1093/ oso/9780198793748.003.0029.

Auer, R. y Boehme, R. (2020). The technology of retail central bank digital currency. Bank for International Settlements. Quarterly Review, March, p. 16. Disponible en: https://bit.ly/3APoP7e.

Auer, R. y Böhme, R. (2021). Central bank digital currency: the quest for minimally invasive technology. Basilea: Bank for International Settlements. Disponible en: https://bit.ly/2Z0VOsB.

Auer, R., Cornelli, G. y Frost, J. (2020). Rise of the central bank digital currencies: drivers, approaches and technologies. CESifo Working Paper, 8655. Disponible en: https://bit.ly/2Xpmr9x.

Beckmann, J., Gern, K.-J. y Jannsen, N. (2021). Should They Stay or Should They Go? Negative Interest Rate Policies Under Review. European Parliament. Disponible en: https://bit.ly/3phBkXg.

Bindseil, U. (2020). Tiered Central Bank Digital Currency and the financial system. European Central Bank. Disponible en: https://bit.ly/3namlvg .

Bindseil, U. (2021). Issuing a digital euro. En European System of Central Banks and International Organisations. European Central Bank. Legal Conference 2020 (pp. 172-178). Disponible en: https://bit.ly/3BXG9Iv.

Bjerg, O. (2017). Designing new money - the policy trilemma of central bank digital currency. Disponible en: https://doi:10.2139/ssrn.2985381.

Boar, C. y Wehrli, A. (2021). Ready, steady, go? Results of the third Bank for International Settlements survey on central bank digital currency. Disponible en: https:// bit.ly/3C2HApm.

Bonatti, L., Fracasso, A. y Tamborini, R. (2021). Unconventional Policy Instruments and Transmission Channels: A State-Contingent Toolbox for the European Central Bank. Department of Economics and Management, 05. Disponible en: https://bit.ly/3aTnXns.

Brunnermeier, M., James, H. y Landau, J.-P. (2019). The Digitalization of Money. National Bureau of Economic Research, 26300. Disponible en: https:// doi:10.3386/w26300.

Carstens, A., Claessens, S., Restoy, F. y Shin, H. S. (2021). Regulating big techs in finance. Hyun Song, Regulating big techs in finance. Disponible en: https://doi. org/10.2139/ssrn.3901736 
Cœuré, B., Cunliffe, J., Nihon Ginkō, Sveriges Riksbank, Schweizerische Nationalbank, Bank of England, Federal Reserve System y Board of Governors. (2020). Central bank digital currencies: foundational principles and core features report no. 1. In a series of collaborations from a group of central banks. Disponible en: https://bit.ly/3DRewS7.

Dowd, K. (2018). Against helicopter money. Cato Journal, 38 (1), 147-170.

Fasquelle, V. (2021). Central Bank Digital Currency: how central banks approach innovation. En Eurosystem (ed.). European System of Central Banks (pp. 179-190). Frankfurt am Main: European Central Bank.

Gortsos, C. V. (2020). The Basic Tasks of the European Central Bank Within the Eurosystem and Issuance of Banknotes and Coins. En European Central Banking Law (pp. 281-329). Cham: Palgrave Macmillan. Disponible en: https://doi:10.1007/978-3-030-34564-8_7.

Gros, D. y Alcidi, C. (2019). Twenty years of the euro-Resilience in the face of unexpected challenges. Monetary Dialogue. Centre for European Policy Studies. Special Report.

Grünewald, S., Zellweger-Gutknecht, C. y Geva, B. (2021). Digital euro and European Central Bank powers. Forthcoming in Common Market Law Review, 58 (4), 1029-1056. Disponible en: https://doi.org/10.2139/ssrn.3807855.

Hinojosa Martínez, L. M. (2021). Euro digital o criptoeuro: ¿Está en juego la soberanía monetaria europea? Revista de Derecho Comunitario Europeo, 25 (69), 471-508. Disponible en: https://doi:10.18042/cepc/rdce.69.01.

Kirchhof, P. (2021). Geld im sog der negativzinsen. München: C.H. Beck.

Knapp, G. F. (1923). Staatliche theorie des geldes. Berlin: Duncker und Humblot. Disponible en: https://doi.org/10.3790/978-3-428-56561-0.

König, J. (2017). Bargeld als Garant für Freiheit und Eigentum. List Forum für Wirtschafts- und Finanzpolitik, 42 (4), 341-364. Disponible en: https://doi:10.1007/ s41025-017-0056-8.

Larue, L., Fontan, C. y Sandberg, J. (2020). The promises and perils of central bank digital currencies. Revue de la Régulation. Capitalisme, Institutions, Pouvoirs, 28. Disponible en: https://doi:10.4000/regulation.18018.

López Escudero, M. (2017). La Unión Económica y Monetaria. En VV. AA. Las políticas de la Unión Europea en el Siglo XXI (tomo II, pp. 339-37). Barcelona: Editorial Bosch. Disponible en: https://doi:10.2307/j.ctvr33bnv.

Lupo-Pasini, F. (2021). Financial Inclusion and the War for Cash. Law and Contemporary Problems, 84 (1), 17-34.

Mancini-Griffoli, T., Peria, M. S. M., Agur, I., Ari, A., Kiff, J., Popescu, A. y Rochon, C. (2018). Casting light on Central Bank Digital Currency. International Monetary Fund Staff Discussion Note, 8. Disponible en: https:/doi:10.1093/ oso/9780190077310.003.0012.

Mersch, Y. (2019). Money and private currencies: reflections on Libra. Twenty Years of Building Bridges: The Process of Legalisation of European Central Banking, 15. 
Murphy, C. (2016). Pecunia Non Olet: The Case for the $€ 500$ Banknote. European Journal of Crime, Criminal Law and Criminal Justice, 24 (4), 340-353. Disponible en: https://doi:10.1163/15718174-24042100.

Nabilou, H. (2020). Testing the waters of the Rubicon: the European Central Bank and central bank digital currencies. Journal of Banking Regulation, 21 (4), 299-314. Disponible en: doi:10.1057/s41261-019-00112-1.

Papapaschalis, P. (2021). Retail central bank digital currency: a (legal) novelty? European Central Bank, 351.

Parra, A. M. (2018). Dinero electrónico revisitado. Revista de Derecho Bancario y Bursátil. Centro de Documentación Bancaria y Bursátil, 37 (151), 9-60.

Siekmann, H. (2020). Monetary Aspects of the Euro as Single European Currency - a German Perspective. En R. Freitag y S. Omlor (eds.). The Euro as Legal Tender (pp. 1-50). Berlin: De Gruyter. Disponible en: https:// doi:10.1515/9783110640717-001.

Strauss-Kahn, M.-O. (2021). A Digital Euro. Atlantic Council [blog], 22-8-2021. Disponible en: https://bit.ly/3CXIYLd. 